\title{
Deriving policies from connection codes to ensure ongoing voltage stability
}

David Ryan ${ }^{1 *}$, Miguel Ponce De Leon ${ }^{1}$, Niall Grant ${ }^{1}$, Bernard Butler ${ }^{1}$, Steffen Vogel ${ }^{3}$, Markus Mirz ${ }^{3}$ and Pádraig Lyons ${ }^{2}$

From The 8th DACH+ Conference on Energy Informatics,

Salzburg, Austria. 26-27 September, 2019

\author{
*Correspondence: dryan@tssg.org \\ ${ }^{1}$ Telecommunications Software \\ Systems Group, Waterford Institute \\ of Technology, Carriganore, \\ Waterford, Ireland \\ Full list of author information is \\ available at the end of the article
}

\begin{abstract}
The management of distribution and transmission networks is becoming increasingly complex due to the proliferation of renewables-based distributed energy resources (DER). Existing control systems for DER are based on static specifications from interdependent network connection documents. Such systems are inflexible and their maintenance requires concerted effort between grid stakeholders.

In this paper we present a new supplementary control approach to increase the agility of the electricity grid. The ICT system that underlies smart grids has the potential to offer, by analogy with ICT based network management, a control plane overlay for the modern smart grid. Policy-based Network Management (PBNM) is widely deployed in managed telecoms networks. We outline how PBNM can augment the management of power and energy networks and report on our initial work to validate the approach. To configure the PBNM system, we have used text mining to derive connection parameters at the LV level. In our simulations, PBNM was used in collaboration with a Volt-VAr optimisation (WO) to tune the connection settings at each DER to manage the voltage across all the buses. We argue that the full benefits will be realised when stakeholders focus on agreeing relatively stable high-level connection policies, the policies being refined dynamically, and algorithms such as WO that set connection parameters so they are consistent with those high-level policies. Thus faults, power quality issues and regulatory infringement can be identified sooner, and power flow can be optimised.
\end{abstract}

Keywords: Smart grid, Text mining, Voltage stability, Electrical engineering, Policy based network management, Grid codes

\section{Introduction}

The electrical energy generation and distribution system is changing. The goal of reducing $\mathrm{CO}_{2}$ emissions and the emergence of DER provide new electrical engineering and regulatory challenges. These challenges, in particular, the large-scale proliferation of renewables-based DER embedded in the distribution network, present challenges to distribution system operators (DSOs) in terms of the cost-effective management of voltage and thermal congestion (Jiayi et al. 2008). One approach is the use of Volt-VAr Optimisation (VVO) algorithms to manage voltage levels and reactive power in the presence of a mix of both traditional and significant renewable energy sources. While this 
improves grid efficiency by stabilising voltage levels, the use of VVO has the potential to circumvent the rules and regulations specified in grid codes and standards. Therefore, there is a risk that the setpoints altered for operational reasons by VVO algorithms might fall outside the ranges specified for such parameters in the formally agreed Distribution Codes.

Currently the monitoring of DER, with respect to distribution codes and standards, is carried out manually and retrospectively. Given that VVO algorithm trials are under way, validating their outputs against the relevant Distribution Codes needs to be moved closer to where those algorithms are used. Therefore we investigate how well-established ICT techniques like

- Text Mining (to extract the critical parameter settings from the Distribution codes), and

- Policy Based Network Management (PBNM, used here to ensure that global interconnection settings mandated in the Distribution Codes continue to apply)

can help. Otherwise, grid control systems need to rely on manual intervention to ensure that VVO algorithms can continue to ensure local voltage stability, while also meeting the global interconnection settings mandated in the Distribution Codes.

This paper is organised as follows. The "Background" section describes the problem domain: the smart grid as a complex system of interacting components ("Smart Grid" section), both power- and ICT-related; the role of regulatory network codes in enabling this complexity of supply ("Network Codes" section); the algorithms that are needed to ensure stable voltage when connecting small-scale renewable energy supplies to the grid ("Volt-VAr Optimisation" section), and finally some techniques like PBNM that are used in other types of networks and might be helpful in the smart grid ("Policy Based Network Management (PBNM)" section). The "Methodology" section describes our initial experience deriving machine-readable configuration settings from typical network code documents ("Network Code Preparation" section), real-time simulations to validate the active voltage management (AVM) control algorithm and to show how this can be placed in a PBNM framework ("AVM Simulation and PBNM Implementation" section). "Future Work" section describes how proposed enhancements of our text mining code could be used to verify the conformity of sets of network code documents ("Network Code and Standards Semantic Alignment" section), and this could be used to ensure that the smart grid is robust, delivers stable voltage and enables greatly enhanced self-management of the energy grid ("Smart Grid and Policy Based Network Management" section). We end with a "Conclusions" section section, which summarises our findings and proposal.

\section{Background}

Policy-Based Network Management (PBNM) and Text Mining are established tools in the ICT domain, but we believe the main contribution of this paper is how they can help to ensure stable voltage profiles in a power and energy market that has many Distributed Energy Resources (DERs). In this section we introduce these concepts and place them in the context of power and energy domain concerns such as Volt-VAr Optimisation (VVO), the smart grid and EU Network Codes (EUNC). 


\section{Smart Grid}

The smart grid is an electrical system that uses information, two-way, cyber-secure communication technologies, and computational intelligence in an integrated fashion across the entire spectrum of the electrical energy system from generation to consumption of electricity (Gharavi and Ghafurian 2011). The traditional paradigm is a unidirectional flow of power from centralised generation units, through the transmission system to the distribution system and ending with the consumer. The move towards de-carbonisation promotes the emergence of low carbon methods of energy generation and consumption and requires a more distributed energy system, characterised by bidirectional flows of energy and ICT communications and providing flexibility to integrate renewables based DER. This paradigm changes the potential to reduce inefficiencies in traditional power systems, like low fuel to power conversion rates and an over reliance on peak time generation over non peak time storage for peak time use (Farhangi 2010).

\section{Network Codes}

Network Codes, also known as interconnection guidelines or connection codes, specify the technical and operational characteristic requirements of power plants and other parties involved in the production, transportation and utilisation of electric power. Such codes first appeared in the USA in the 1990's after the de-regulation of the generation of electric power and have since been extended to include all the main entities in the power supply chain (Kumar and Singh 2012).

With de-regulation has come new markets, actors and business models, and Network Codes are needed to support such new developments. Recent trends include the growth of Peer2Peer energy trading, new entrants to the markets like aggregators, new entities like microgrids and the emergence of new generation concepts like micro-generation and DER (Guerrero et al. 2010). Consequently, the role of Network Codes has broadened.

Meanwhile ICT and advanced communications facilitate control and other data flows (including usage) in parallel with the supply concerns addressed in the Network Codes. At present, Network Code-based control and smart grid ICT systems have limited interoperability, largely due to the fact that the Network Codes are printed documents governing each link in the energy supply chain. Consequently, they require expert (human) interpretation to resolve any discrepancies and are not easy to integrate with the electronic data flows elsewhere in the smart grid.

\section{Volt-VAr Optimisation}

As more renewable-based DERs offer electricity to the grid, decentralised control becomes more important. Typically DER (solar, wind and energy storage) are coupled to the distribution network using power electronic based inverters. The inverter settings need to be chosen carefully, to convert some of the DER's energy output to reactive power, but only just as much as is needed for voltage stability in the DSO. Therefore greater control efficiency can reduce energy losses where this power enters the electricity supply network. This is good for the environment and also increases the DSO's profits (Civanlar and Grainger 1985).

An output of the RE-SERVE project, which funded this study, is the deployment of an Optimal Power Flow (OPF) (Carpentier 1962) derived method, known as Active Voltage 
Management (AVM), to edge DER components in an effort to increase voltage stability and reduce energy losses. This method has two stages, the first is off-line modelling of the steady state of the network to determine the optimal voltage using the 3-OPF tool (Bakhtvar et al. 2017), producing a Volt-VAr curve (VVC). In the second stage the VVC is deployed to a real-time controller on the RES unit.

We note that other VVO algorithms are available. Wang et al (2017) (Wang et al. 2017) categorise voltage control algorithms as either deterministic (such as the oriented discrete coordinate decent method (ODCDM)) or meta-heuristic (such as Cuckoo Search (Yang and Deb 2009)). Other meta-heuristic VVO techniques listed by Keane et al. (2013) include robust search techniques such as ant colony optimization (ACO), artificial bee colony optimization (ABC), tabu search (TS), particle swarm optimization (PSO), simulated annealing (SA) and genetic algorithms (GA). In principle, any of these techniques could be used instead of AVM in our study.

\section{Policy Based Network Management (PBNM)}

As the electrical power industry shifts from being centralised to a more distributed generation and distribution model, ICT support has become essential. ICT can assist with core operations, billing and demand prediction (Bakhtvar et al. 2017). Such applications require (data) network connectivity (typically a dedicated network or a virtual private network over the public internet) between all interconnected devices in the energy network, collectively known as the smart grid. As an example of critical network infrastructure, the smart grid has security, latency and entity control requirements (Gungor et al. 2011) that are similar to critical ICT infrastructures in other domains (such as telecommunications and enterprise data networks) where PBNM has proved useful (Strassner 2003).

At its core, PBNM decouples the policies (i.e., the rules that the system needs to satisfy, together with their context) from how those rules are applied (Sloman 1994). This separation of concerns introduces great flexibility and provides opportunities to be more efficient to implement. The PBNM infrastructure includes standard components such as Policy Administration Points (PAP: where policies are written), Policy Decision Points (PDP: where a policy is consulted and a decision returned) and Policy Execution Points (PEP: where requests for context changes are intercepted, passed to a PDP, and its decision is returned to the requesting entity) (Durham et al. 2000).

Furthermore, PBNM includes the concept of a policy continuum, where high-level (hence less detailed and more abstract) policies can be refined with the aid of an information model to low-level (fully detailed, hence deployable to devices) policies (Davy et al. 2007). Therefore, with appropriate policy authoring tools and support, policies can be more dynamic and responsive to local changes in their environment.

The off-line analysis involving AVM and VVC optimisation described in the subsection on "Volt-VAr Optimisation" section is a limitation, particularly when there are many RES devices in the model. A large number of DER sources can lead to a loss of VVC consistency and relevance because of the lack of VVC updating mechanisms at a device level. These limitations in the operation of the AVM pose challenges but AVM is still viable because PBNM could help to ensure that each AVM instance meets global Network Code constraints. This can happen if the off-line generation of the VVC is performed by a specialised PAP and deployed as a policy. By combining the policy with contextual information (in the form of the measured state of the network) in the PDP (where the policy 
is applied), it is possible for the energy system to respond dynamically to changes in the network state. This is for the same reasons that PBNM enables ICT systems like cloud computing to respond to changes in workload, while also satisfying service level agreements (encoded as policies) between cloud infrastructure/platform/software providers and their clients.

\section{Methodology}

This section describes the implementation of a VVO technique, Active Voltage Management (AVM), in a real-time simulation environment. The objective is to demonstrate how ICT techniques, like PBNM and Text Mining, can prepare the connection codes and use them as policies to configure and set constraints within voltage optimization algorithms.

\section{Network Code Preparation}

To use the connection codes for PBNM, it is necessary to extract the quantifiable computer-readable values from the network code documents. To do this we first need to derive the structure of each document, as this helps to identify candidate text for further processing, especially when it might contain similar concepts in different connection code documents.

We then parse the candidate text into values and supplementary text. The values are numeric parameters that control the distribution and transmission of power and the supplementary text provides context to the values so that they can be interpreted by humans. The large number of values, their interdependence with other values in a given document (moderated by the context provided by the supplementary text), and the relationships between values in different documents, combine to make this a challenging problem.

Therefore, prior to the use of the VVO algorithm to validate the PBNM approach, it is important to extract all the relevant values from all the versions of network code policy documents. This can be achieved in two steps:

- text mining to normalise the text and extract the relevant parameters, followed by

- concept modelling to compare all extracted versions and create a combined (federated) version of the applicable grid codes.

The outputs of these stages contain a computer-readable set of values to be deployed within the AVM algorithm. As an example of this process, the data in Table 1 was extracted from a connection code document and converted to the computer-readable snippet in Fig. 1.

The extracted parameters are now presented in a computer readable format (see Fig. 1), but the candidate text is in a human written format. Thus we must check whether text mining captures the meaning of the settings and not just the settings themselves, so that they can be interpreted correctly. This is a more difficult task, requiring high accuracy, because we intend to deploy these policies on critical systems in the grid. In

Table 1 Power Factor - Irish Distribution Code Example

\begin{tabular}{llll}
\hline Voltage Range & Connected at: & At 100\% Registered Capacity & At 35\% of Registered Capacity \\
\hline $99 \mathrm{kV} \leq V \geq 123 \mathrm{kV}$ & $110 \mathrm{kV}$ & $\begin{array}{l}0.93 \text { power factor leading to 0.85 } \\
\text { power factor lagging }\end{array}$ & $\begin{array}{l}0.7 \text { power factor leading to 0.4 } \\
\text { power factor lagging }\end{array}$ \\
$85 \mathrm{kV} \leq V \geq 99 \mathrm{kV}$ & $110 \mathrm{kV}$ & $\begin{array}{l}\text { Unity power factor to 0.85 power } \\
\text { factor lagging }\end{array}$ & $\begin{array}{l}0.7 \text { power factor leading to 0.4 } \\
\text { power factor lagging }\end{array}$ \\
\hline
\end{tabular}




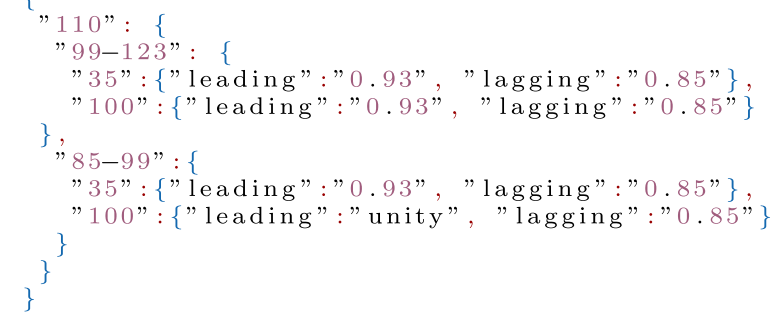

Fig. 1 Connection settings, in JSON (Crockford 2006) format, consistent with Table 1

the present state of the art, these network codes are interpreted manually by human experts. If computers are expected to read such documents in production deployments, the outputs should be validated against domain-specific standards, specially designed for such validation tasks. There are precedents in other domains. For example, computer interpretation of written standards is also a feature of biomedicine, so the stakeholders there have developed "Gold Standard" corpora (terms, their usage and meaning) like GENIA (Kim et al. 2003) and PennBioIE (Liberman et al. 2008), as mentioned by (Ananiadou et al. 2006). Given such text corpora, users can derive statistical analysis scores that measure the accuracy of various text mining approaches.

In the absence of an agreed connection code corpus for the electrical grid, we checked the extracted parameters manually. According to this validation, limited to a small set of connection code documents, we found that text mining was accurate but acknowledge that this approach is not sufficient to make general claims about the quality of our approach. Therefore, in the absence of a standard corpus of connection codes, we recommend that human experts continue to inspect the outputs of text mining, but that work should proceed on gathering such a corpus to help validate the computer interpretation of connection code documents. In particular, where techniques struggle to "read" some settings correctly, those settings (and their representation) should be added to the corpus, thereby increasing its coverage.

In this worked example, we use Ireland's national Grid Code (EirGrid and Grid Code v6 2015) and Distribution Code (ESB 2016) to demonstrate how PBNM can modify the VVO constraints in an efficient and timely manner.

Usually, text mining is used in applications like sentiment analysis to derive summaries of massive volumes of data (such as tweets). In this paper, we propose applying such techniques to much smaller volumes of much more dense, semi-structured data, searching for specific information regarding connection settings. Our aim is to meet the challenge laid out by Gungor et al. (2011), where they state that interoperability is a critical prerequisite for the mass adoption of new renewable DERs, to integrate them successfully into the existing grid. As the grid become more heterogeneous and needs to respond more dynamically, connection codes need to be interpreted and checked more frequently. Without a technique such as text mining, the limited availability of human experts could act as a bottleneck to the continued expansion and growth in flexibility of the electrical grid. Therefore, we argue that there is an acute need for a connection code corpus, and the supporting agreements and infrastructure. Also, we note that the connection codes 
form a small but significant part of how the electrical grid is regulated, so it might be possible to widen the scope to include a more comprehensive set of the regulatory guidelines in the future.

\section{AVM Simulation and PBNM Implementation}

To demonstrate the capability of PBNM to ensure that connection code constraints are respected in VVO, we evaluate its impact on a single parameter, power factor, in a real-time simulation. These simulations were performed by partners in the RESERVE project to validate the operation of the chosen VVO algorithm (AVM). The simulations used an Opal-RT real-time simulator to model a 3-phase LV network model, see Fig. 2, containing a mixture of three-phase and single-phase buses with a 3kVA vehicle-to-grid (V2G) electric vehicle (EV) charge point. A V2G EV chargepoint is capable of both importing energy from the distribution network to charge a vehicle but is also capable of discharging the vehicle battery, exporting energy to the distribution network. While PBNM was not included in the simulation, we note the analogies:

- The Volt-Var curve produced by AVM is equivalent to a policy,

- the control actions that result are equivalent to the decisions made by the policy,

- the changing power state is equivalent to the context that is part of the policy decision,

- measurements of reactive power or voltage provide the monitoring data to enable autonomous PBNM operation.

Figure 3 shows a voltage control scenario designed to minimize voltage imbalance, where the power factor values, primarily the Unity Power Factor, are considered as constraints when generating the VVC. The sequence of events in Fig. 3 show the percentage of voltage imbalance on each bus with 4 events occurring 1 . Charger enabled at $0 \mathrm{~s}, 2$. AVM enabled at $100 \mathrm{~s}, 3$. AVM disabled at 500s and 4. simulation ends at $600 \mathrm{~s}$.

In the creation of the VVC the algorithm used unity power factor as an upper bound which ensured that the power quality remained at an acceptable level. The plot in Fig. 4 demonstrates this and while the goal of the simulation was to ensure voltage stability the unity power factor constraint was obeyed to also show consideration for power quality. In the development of the VVC for this simulation, however, the lagging power factor was not considered as a bound but the application of PBNM could address this by monitoring the power quality using relevant policies to detect any extreme violations and instructing the VVC generation to apply a lagging power factor constraint.

The simulation has shown that AVM succeeded in providing voltage stability and that the Unity Power Factor ensured adequate power quality. This simulation was a proof of concept: it used just a single connection code constraint (i.e., the Unity Power Factor bound). In practice, many more regulatory constraints would need to be enforced and this would make VVO algorithm configuration much more difficult.

PBNM, supported by text mining to derive suitable parameter ranges, potentially makes this much easier, because it scales more easily as the number of connection code constraints increase. PBNM also offers a more transparent way to view the operation of the control system, since it was designed by (data) network engineers to be analogous to traditional closed-loop control systems such as are used here. 


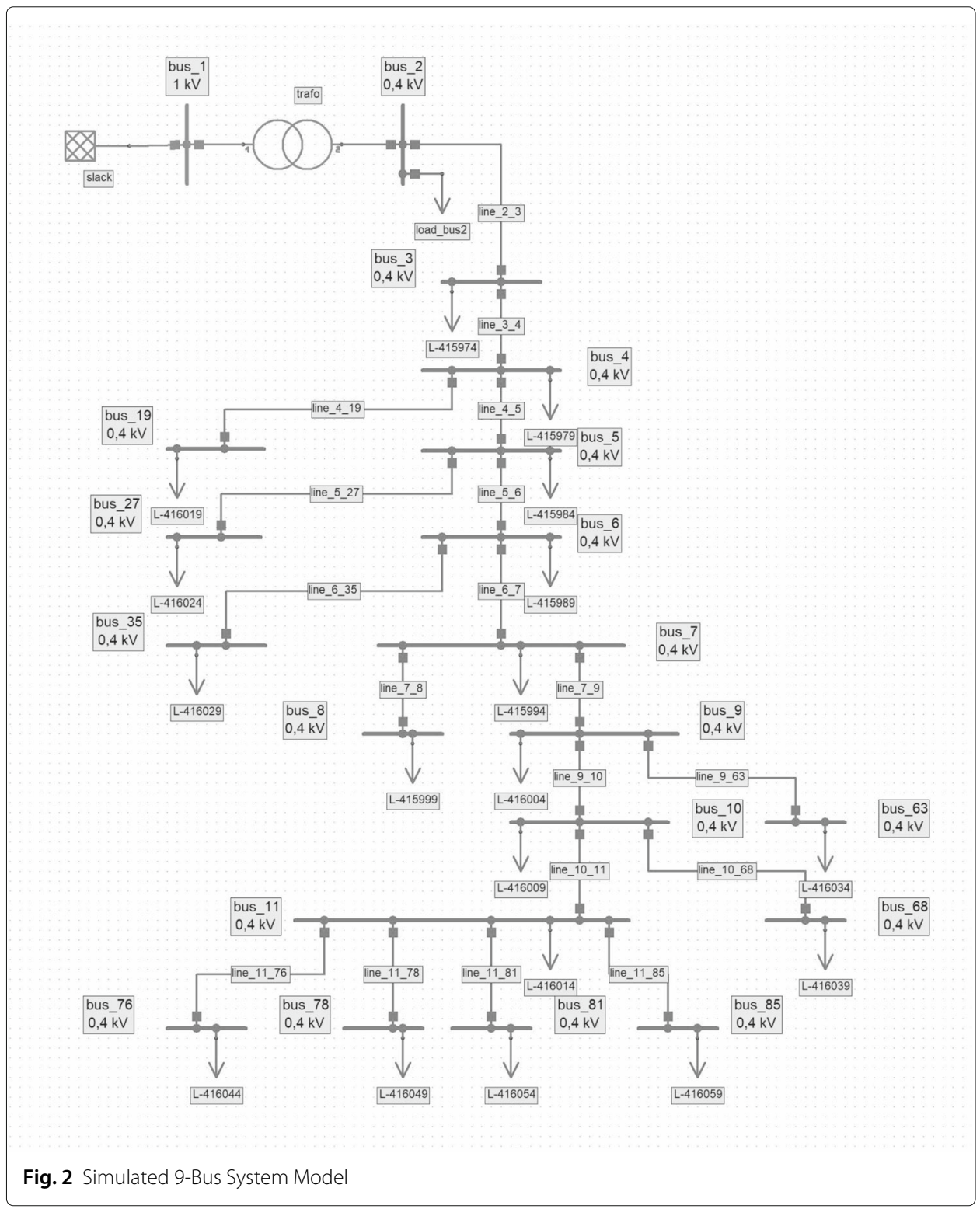

Figure 5 illustrates a scenario where an AVM-PBNM system operates on the data with both a compliant and non-compliant reactive power set point.

The main advantage of PBNM in this context is the opportunity to ingest network codes dynamically, as the power network configuration changes, without the need for complex service agreement setting between stakeholders. The various stakeholders still need to a) provide specifications and ensure their devices continue to meet them, and b) to specify the interconnection (high-level) guidelines but PBNM, with suitable VVO algorithms, can take responsibility for managing the set-points that control the network.

To demonstrate this, a second experiment was performed where the active power dropped to one tenth of what was expected and, as a result, the power factor dropped below its acceptable level. Figure 6 illustrates the drop in Active Power taking place at 14:53 and hence the VVC used to achieve stability is no longer sufficient. This change in 


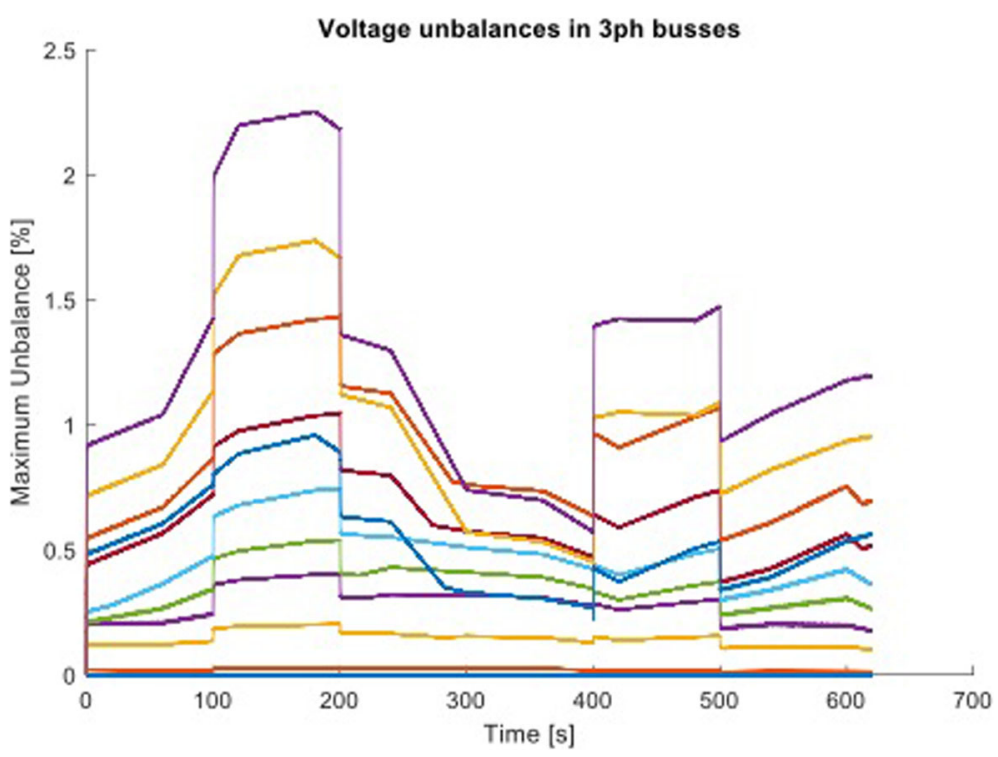

Fig. 3 Voltage Readings from 9-Bus V2G System

network state is unacceptable so the PDP triggers a policy that instructs the AVM component to create an updated VVC. The new VVC is deployed at the RES at 14:58, thereby restoring voltage stability. Meanwhile the bounds applied to the generation of the VVC can be maintained to ensure that the Power Factor does not exceed the Leading Power Factor ensuring acceptable voltage quality.

For this paper, the policy was checked manually and the call to the AVM module to generate a new VVC was also manual. However, if this were to be implemented using PBNM, the overall procedure would remain the same. Figure 7 indicates how this scenario would

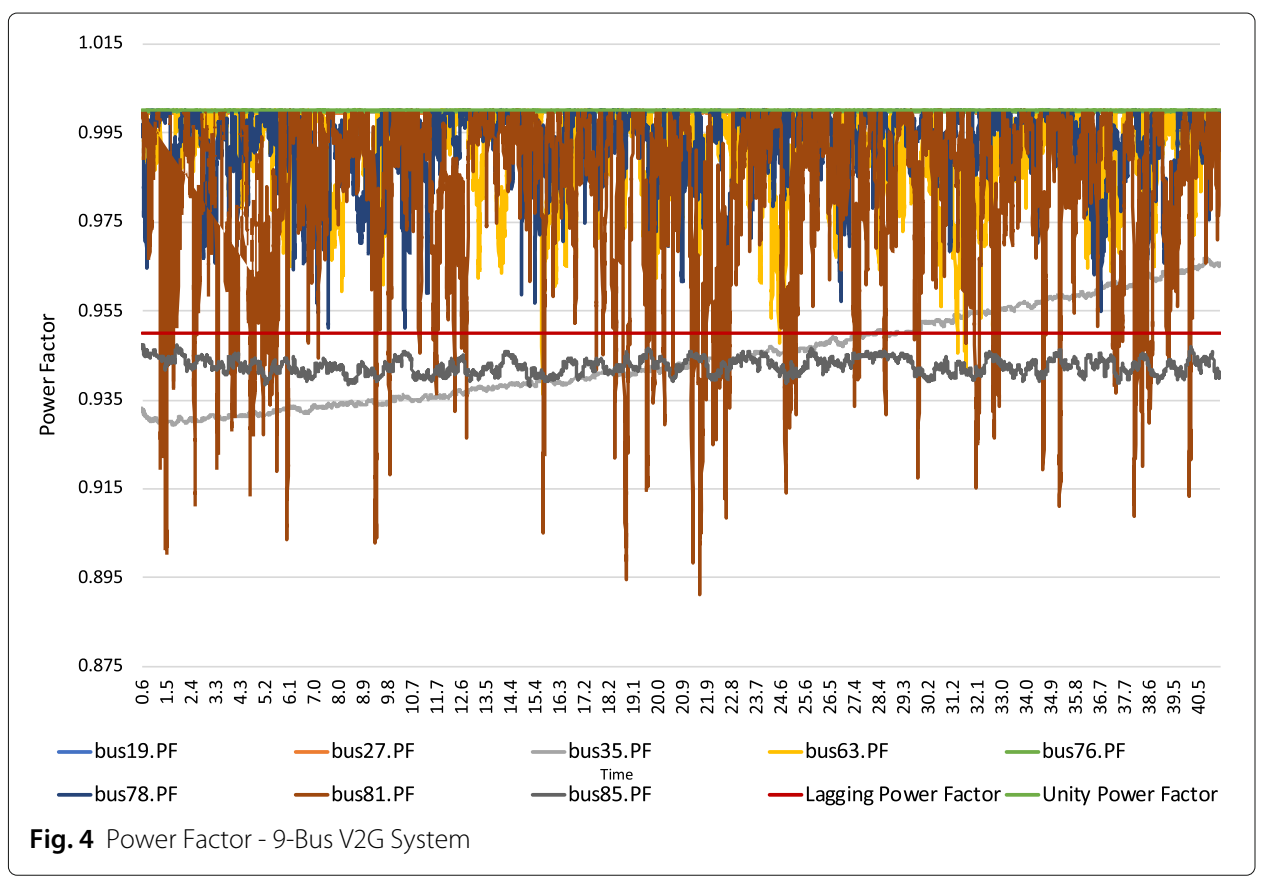




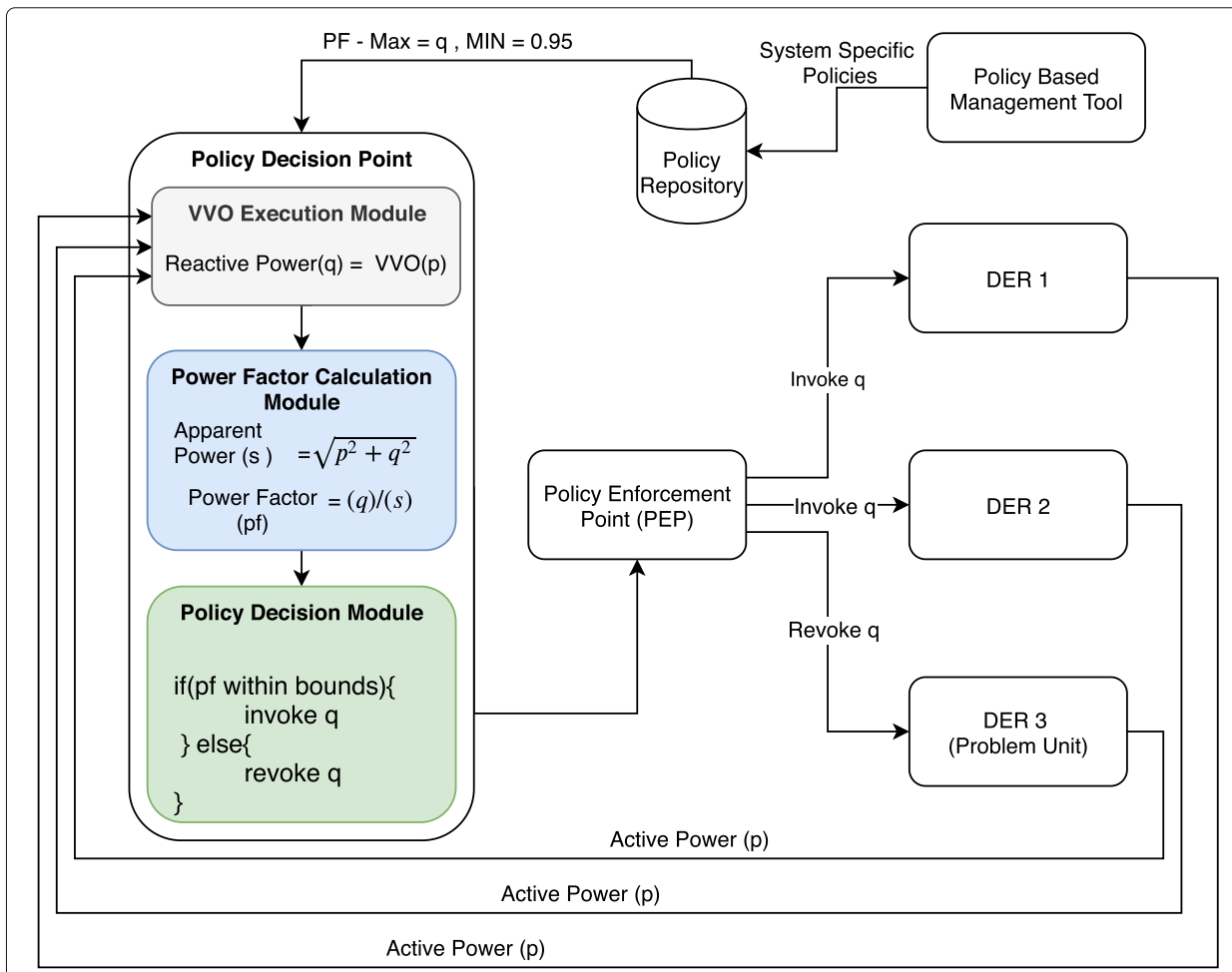

Fig. 5 Policy Based Network Management Voltage Control Validation

be implemented using PBNM. The PDP would validate the Power Factor (PF) against the relevant policy supplied by the policy database and, if the bounds are violated, the system would trigger the policy obligation within the policy to request an updated VVC from the 3-OPF tool. One more check would then take place to verify the PF against the policy to determine if the issue has been resolved by this operation. If not, the updated VVC would

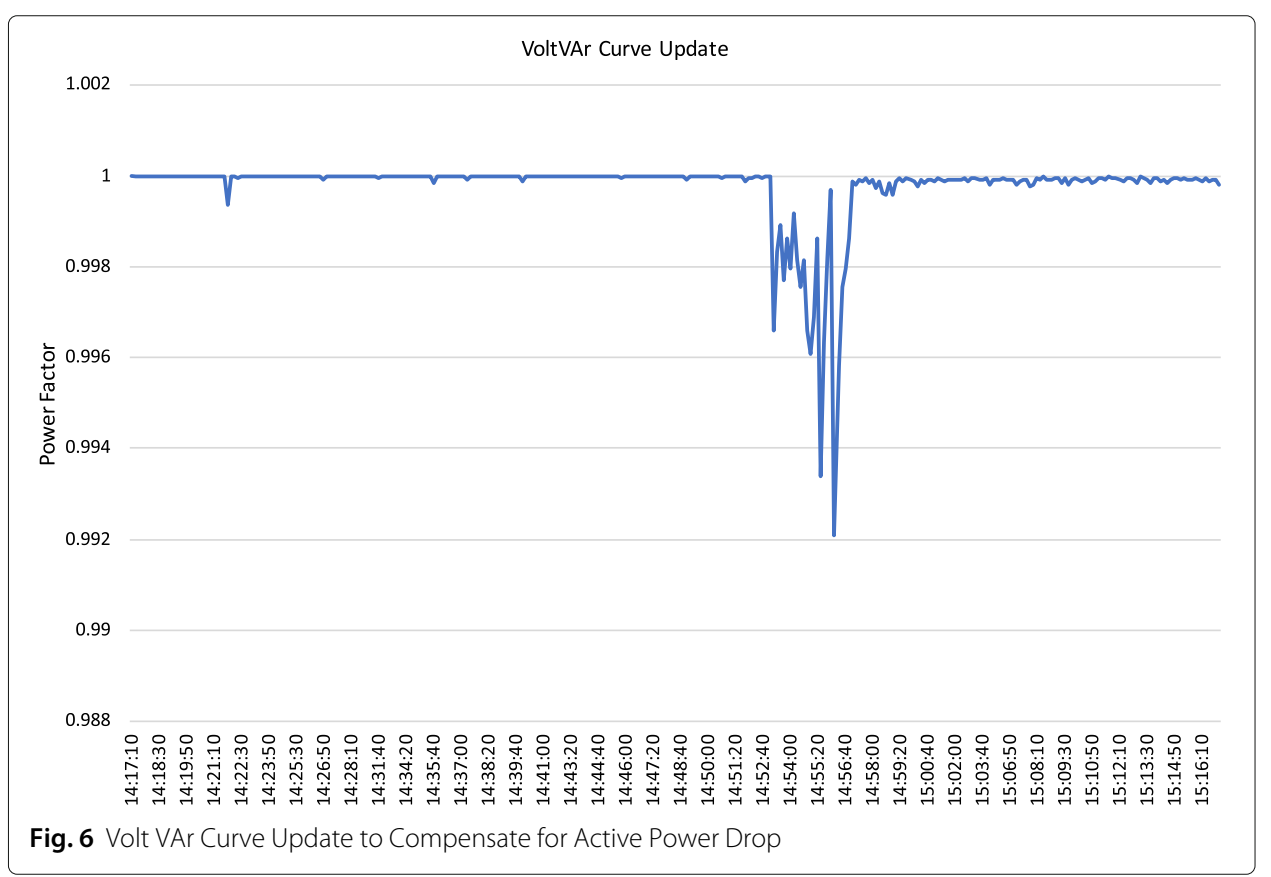




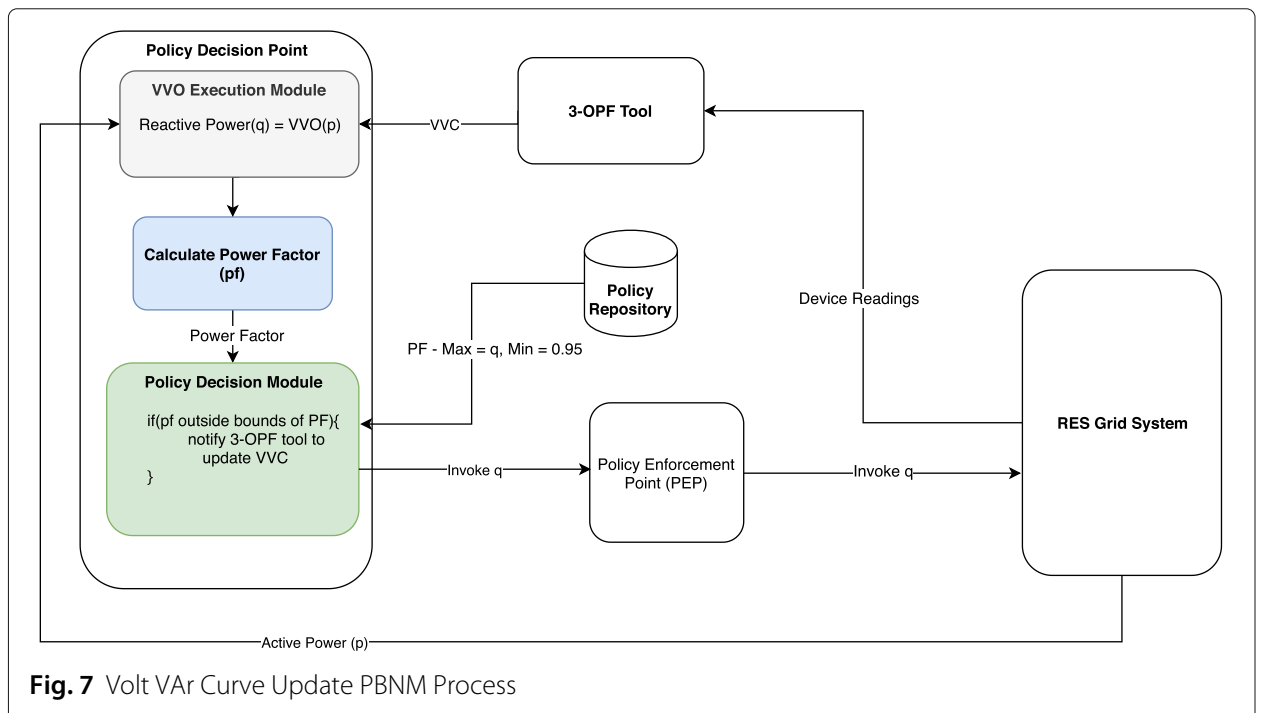

be used to calculate the reactive power set point to be sent to the inverter to stabilise the Power Factor. One of the advantages of the combined AVM-PBNM approach is that the current manual process could be automated, so voltage stability could potentially be restored much more quickly, and not in 5 minutes as is the case in this test.

Furthermore, recall that AVM deploys VVCs to the managed DER devices to improve their voltage stability at a given instant. However, without an effective VVC update mechanism, demand response factors and network properties might change dynamically, thereby invalidating some of these VVCs. In such scenarios, PBNM could inform the AVM of those changes, triggering it to generate and deploy more relevant VVCs to the relevant DERs. Thus PBNM would help AVM to maintain voltage stability even when the grid becomes more dynamic.

\section{Future Work}

This paper presents early-stage research into the use of PBNM and text mining to solve practical voltage control problems in power and energy systems. We have shown (by simulation in subsection "AVM Simulation and PBNM Implementation" section) that AVM can apply the necessary control operations and have discussed in the subsection on "Network Code Preparation" section how PBNM and text mining can overcome the problems of static control definitions, and have indicated in Fig. 5 how they can be brought together into a practical framework.

The subsections "Smart Grid and Policy Based Network Management" section and "Network Code and Standards Semantic Alignment" section below discuss the two potential applications of ICT techniques in the electricity supply industry.

\section{Smart Grid and Policy Based Network Management}

In previous sections we have demonstrated how PBMN systems could be applied to dynamically apply constraints to the operation of a VVO algorithm like AVM, by incorporating it in a framework that makes it easier to apply connection code constraints in a more dynamic fashion. However PBNM could also help to manage the ICT component of the smart grid, not just the power and energy elements. 
Such use-cases would be underpinned by the bi-directional communications in a Smart Grid:

- downstream propagation of rules and policies to devices throughout the power grid, and

- upstream sending of alerts and reporting to controllers and decision support systems, and

- bidirectional sending of device to device control messages and actions in a distributed power system.

When smart grid monitoring, weather and market data are available, the control aspects of PBNM could be augmented with machine learning and similar technologies to support more complex use cases like

- Autonomics and Self Healing,

- Fault and Power Quality Alerting,

- Regulatory Infringement Alerting,

- Low Level Device Access and Visibility, and

- Power Flow Optimisation.

\section{Network Code and Standards Semantic Alignment}

As outlined in the "Background" subsection on "Network Codes" subsection, there are many challenges when it comes to the regulation (e.g., for stability of supply) and management (e.g., for energy efficiency) of power generation and distribution systems. Such challenges include the emergence of renewables-based DER, the decommissioning of large fossil fuel plant, the emergence of domestic and commercial customers as active participants, and the electrification of the transport and heat sectors. The system requires far greater flexibility to maintain reliable supply, and the ICT techniques described in this paper and being considered in the RE-SERVE (RE SERVE (H2020 funded Grant Agreement no. 727481) 2017) project could result in extensions to the existing network code infrastructure.

One of our findings is the need for semantic alignment across all connection codes, at least per geographical region (e.g., a single large country or a group of interconnected countries). Semantic alignment ensures that connection codes are interpreted and applied consistently by all stakeholders. It would facilitate the extraction of key quantities in a form that could be deployed in more agile forms of control, such as a PBNM-inspired infrastructure for a smart grid. The benefits of such agility include easier modification and updates to the connection codes themselves, and their traceable deployment to all the relevant devices in the grid. Future work would use text mining to extract and normalise the quantifiable values and use Natural Language Processing to enable the mapping of different codes across various versions of connection code documentation.

\section{Conclusions}

This paper looked at smart grid deployment of regulatory network codes, especially where they pertain to DERs. It addressed the specific issue of the use of VVO algorithms like AVM to ensure the stability of voltage in the DSO and by extension in the wider electrical grid. Because the monitoring of network codes can be done only manually and retrospectively some minutes, hours, weeks and even months after an event, we report on 
the validation of the current state of the art: an online voltage control algorithm (AVM) but with offline setting of the underlying connection parameters. Such offline configuration processes are time-consuming and weaken the flexibility of VVO. They need to be addressed if the smart grid is to become more responsive to changes both in supply and demand.

Given the conceptual similarity between the architecture of DER systems and large scale distributed computer systems, we considered how to apply well-established PBNM techniques, originally proposed for telecoms and data networks, to help manage a heterogeneous electricity grid at the LV level.

Having reviewed the national DSO and TSO network code documents for Ireland, we used text mining to derive a set of network code constraint settings, to be applied at the micro-generation and LV control operations level. Within the RE-SERVE project, we then simulated a scenario where a specific VVO algorithm was applied in a LV system that had DER devices deployed within it. The simulation results suggest that AVM, as our VVO algorithm implementation, can achieve voltage stability while considering power quality using PF bounding. However, manual/static configuration of AVM is a limitation in the current state of the art.

We propose that PBNM is a viable solution for such problems, as it was designed as a solution for equivalent problems in the data networking domain, where it is impractical to configure all (computing, storage or networking) nodes manually. We identified many of the potential advantages of this approach and devised a framework (see Fig. 5) in which PBNM and AVM play essential parts. We are currently working on ways to validate this framework, to check whether it delivers the expected benefits for voltage stability control. Our initial experiments suggest that it is possible to derive configuration settings from the network connection code documents but we have not yet had the opportunity to use this to check the semantic alignment of different documents, which is a precursor to having ubiquitous PBNM in the energy grid.

In future work, we have identified the potential for PBNM to be extended, to take account of new data, such as weather forecasts and both historical and predicted demand. Such data could then be used to make the energy grid more efficient and also more reliable against future events affecting supply, demand or both.

Acknowledgement

There are no acknowledgements relevant to this work.

\section{About this supplement}

This article has been published as part of Energy Informatics Volume 2 Supplement 1, 2019: Proceedings of the 8th $\mathrm{DACH}+$ Conference on Energy Informatics. The full contents of the supplement are available online at https:// energyinformatics.springeropen.com/articles/supplements/volume-2-supplement-1

Authors' contributions

The work in this paper is made up of contributions of authors from three organisations. Telecommunications Software Systems Group main contribution to this research was to explore the use of mature ICT concepts like PBNM and Text Mining to supplement the regulatory frameworks like the grid codes and aid the operation of voltage optimisation concepts like the AVM. Institute for Automation of Complex Power Systems provided the simulations and domain specific knowledge as input from the perspectives of power systems and electrical engineering. ESB Networks LTD provided reviews and validation of the proposed solutions, and also contributed to this work with electrical engineering and domain specific regulatory information from the perspective of a large scale energy provider and grid operator. All authors read and approved the final manuscript.

\section{Funding}

This paper is supported by European Union's Horizon 2020 research and innovation programme under grant agreement No 727481, project RE-SERVE (Renewables in a Stable Electric Grid) (RE SERVE (H2020 funded Grant Agreement no. 727481) 2017). Publication of this supplement was funded by Austrian Federal Ministry for Transport, Innovation and Technology. 
Availability of data and materials

At present none of the data sources or materials used in this work are publicly available.

\section{Competing interests}

The authors declare that they have no competing interests.

\section{Author details}

${ }^{1}$ Telecommunications Software Systems Group, Waterford Institute of Technology, Carriganore, Waterford, Ireland. ${ }^{2}$ ESB Networks LTD, Leopardstown, Dublin, Ireland. ${ }^{3}$ Institute for Automation of Complex Power Systems, RWTH Aachen University, Mathieustraße 10, Aachen, Germany.

\section{Published: 23 September 2019}

\section{References}

Ananiadou S, Kell DB, Tsujii J-i (2006) Text mining and its potential applications in systems biology. Trends Biotechnol 24(12):571-579. https://doi.org/10.1016/j.tibtech.2006.10.002

Bakhtvar M, Cabrera CA, Buttitta G, Neu O, Keane A (2017) A study of operation strategy of small scale heat storage devices in residential distribution feeders. In: 2017 IEEE PES Innovative Smart Grid Technologies Conference Europe (ISGT-Europe). pp 1-6. https://doi.org/10.1109/ISGTEurope.2017.8260254

Carpentier J (1962) Contribution a l'etude du dispatching economique. Bulletin de la Societe Francaise des Electriciens $3(1): 431-447$

Civanlar S, Grainger JJ (1985) VoltNar Control on Distribution Systems with Lateral Branches Using Switched Capacitors and Voltage Regulators Part I: The Overall Problem. IEEE Power Eng Rev PER-5(11):54. https://doi.org/10.1109/MPER. 1985.5528389

Crockford D (2006) JSON: The Fat Free Alternative to XML. In: 15th International WWW Conference, Edinburgh. http:// wwwconference.org/www2006/programme/d8.html. Accessed 19 June 2019

Davy S, Jennings B, Strassner J (2007) The policy continuum-a formal model. In: Proceedings of the Second IEEE International Workshop on Modelling Autonomic Communications Environments (MACE 2007). pp 65-79. http:// www.tssg.org/files/archives/2007_MACE_SDavy_Jennings_final.pdf. Accessed 19 June 2019

Durham DF, Boyle JH, Cohen RB, Herzog S, Rajan R, Sastry A (2000) RFC 2748: The COPS (Common Open Policy Service) Protocol. http://tools.ietf.org/html/rfc2748. Accessed 19 June 2019

ESB (2016) Distribution code. https://www.esbnetworks.ie/docs/default-source/publications/distribution-code-v5-0.pdf. Accessed 29 Nov 2018

Farhangi $\mathrm{H}$ (2010) The path of the smart grid. IEEE Power Energy Mag 8(1):18-28. https://doi.org/10.1109/MPE.2009. 934876

Gharavi H, Ghafurian R (2011) Smart grid: The electric energy system of the future. Proc IEEE 99(6):917-921. https://doi. org/10.1109/JPROC.2011.2124210

EirGrid, Grid Code v6 (2015). http://www.eirgridgroup.com/site-files/library/EirGrid/GridCodeVersion6.pdf. Accessed 29 Nov 2018

Gungor VC, Sahin D, Kocak T, Ergut S, Buccella C, Cecati C, Hancke GP (2011) Smart Grid Technologies: Communication Technologies and Standards. IEEE Trans Ind Inform 7(4):529-539. https://doi.org/10.1109/TII.2011.2166794

Guerrero JM, Blaabjerg F, Zhelev T, Hemmes K, Monmasson E, Jemei S, Comech MP, Granadino R, Frau Jl (2010) Distributed generation: Toward a new energy paradigm. IEEE Ind Electron Mag 4(1):52-64. https://doi.org/10.1109/ MIE.2010.935862

Jiayi H, Chuanwen J, Rong X (2008) A review on distributed energy resources and MicroGrid. Renew Sust Energ Rev 12(9):2472-2483. https://doi.org/10.1016/J.RSER.2007.06.004

Keane A, Ochoa LF, Borges CLT, Ault GW, Alarcon-Rodriguez AD, Currie RAF, Pilo F, Dent C, Harrison GP (2013) State-of-the-Art Techniques and Challenges Ahead for Distributed Generation Planning and Optimization. IEEE Trans Power Syst 28(2):1493-1502. https://doi.org/10.1109/TPWRS.2012.2214406

Kim J.-D., Ohta T, Tateisi Y, Tsujii J (2003) GENIA corpus—a semantically annotated corpus for bio-textmining. Bioinformatics 19:180-182. http://dx.doi.org/10.1093/bioinformatics/btg1023. http://oup.prod.sis.lan/bioinformatics/ article-pdf/19/suppl_1/i180/614820/btg1023.pdf. Accessed 19 June 2019

Kumar P, Singh AK (2012) Renewable Energy Desalination. World Bank. https://doi.org/10.1596/978-0-8213-8838-9. http://arxiv.org/abs/https://elibrary.worldbank.org/doi/pdf/10.1596/978-0-8213-8838-9. http://elibrary.worldbank. org/doi/book/10.1596/978-0-8213-8838-9. Accessed 19 June 2019

Liberman M, Mandel M, White P (2008) PennBiolE Oncology 1.0 LDC2008T21. https://catalog.ldc.upenn.edu/ LDC2008T21. Accessed 19 June 2019

RE SERVE (H2020 funded Grant Agreement no. 727481) (2017). https://www.re-serve.eu/

Sloman M (1994) Policy driven management for distributed systems. J Netw Syst Manag 2(4):333-360. https://doi.org/10. 1007/BF02283186

Strassner J (2003) Policy-based Network Management: Solutions for the Next Generation. Morgan Kaufmann Publishers Inc., San Francisco, CA, USA

Wang P, Yi J, Zangiabadi M, Lyons P, Taylor P (2017) Evaluation of voltage control approaches for future smart distribution networks. Energies 10(8). https://doi.org/10.3390/en10081138

Yang X, Deb S (2009) Cuckoo Search via Lévy flights. In: 2009 World Congress on Nature \& Biologically Inspired Computing (NaBIC). pp 210-214. https://doi.org/10.1109/NABIC.2009.5393690

\section{Publisher's Note}

Springer Nature remains neutral with regard to jurisdictional claims in published maps and institutional affiliations. 